\title{
Preliminary findings of the AALIANCE2 Ambient Assisted Living Roadmap
}

\author{
Alessandra Moschetti ${ }^{1}$, Laura Fiorini ${ }^{1}$, Michela Aquilano ${ }^{1}$, Filippo Cavallo ${ }^{1}$ and Paolo \\ Dario $^{1}$
}

\begin{abstract}
The AALIANCE2 Project, funded by the European Commission's ICT Programme within the $7^{\text {th }}$ Framework Programme, aims at identifying the research priorities in the Ambient Assisted Living (AAL) field in Europe and worldwide for the next decades. One of the main objectives of this Project is the development of an AAL Roadmap and Strategic Research Agenda (SRA) that, starting from the needs of the elderly and caregivers, describes the possible next generation of AAL service scenarios, the necessary key enabling technologies (KETs) and the technological, legal and economic requirements necessary for the implementation of the proposed AAL systems. Some of these new AAL scenarios show how technologies, such as robotics and ICT solutions, could be used in senior citizens' daily life activities to maintain their independence and to stay healthy and active in society. At the same time, other scenarios propose new approaches and solutions for caregivers to efficiently support old persons and optimize their work. The Roadmap and the Strategic Research Agenda finally present the future technological challenges to developing the proposed service solutions. This paper provides a short overview of the preliminary version of the AALIANCE2 Roadmap.
\end{abstract}

\section{Introduction}

The population in Europe is rapidly ageing due to increased life expectancy and low birth rates [1]. The growth in the number of elderly people also means that the number of "retirees" will get higher, reaching the point in 2060 where there will be almost one person over 65 for every two persons of working age in Europe [1]. This increase is causing a wider need for services and more responsibility for society both from an economic and a social point of view.

The current concern of most worldwide governments is that the growth in retirees could affect public finances, increasing costs for the society. Moreover, during the past

\footnotetext{
${ }^{1}$ Alessandra Moschetti, Laura Fiorini, Dr. Michela Aquilano, Dr. Filippo Cavallo and Prof. Paolo Dario

The BioRoboticsInstitute, Scuola Superiore Sant'Anna, Viale Rinaldo Piaggio 34, 56025 Pontedera (PI), Italy, e-mail: f.cavallo@sssup.it, a.moschetti@sssup.it
} 
years the European Union, as well as other countries worldwide, has been facing an economic crisis that is involving all parts of the society. So it is necessary to help people stay as healthy and active as possible and give them the opportunity to remain involved in society and in work for longer.

In order to face the current socioeconomic context, a new approach is necessary, and in this context the Ambient Assisted Living (AAL) model is gaining a foothold. The main characteristic of the AAL approach is the use of ICT technological solutions to improve the quality of life of older persons and to optimise socio-medical services. In the past years several AAL services have been developed following this approach. According to this vision, AAL could support elderly people in staying independent and active and help themenjoy more years of healthy life. Technologies could indeed monitor their health, assist them in executing daily life activities andsupport them in maintaining social contacts and being involved in community life. The current studies carried out in this field show the great potentialities and the possible benefits of AAL in the society; however, there are still technological, social and political barriers delaying the exploitation of AAL in communities. For this reason the European Community is financing the AALIANCE2 Project, a coordinated action funded within the ICT Programme of the $7^{\text {th }}$ European Framework Programme, aiming at identifying challenges and research priorities in the AAL field over the next decades that could allow and facilitate AAL exploitation and deployment of the actual services. In particular, one of the main objectives of AALIANCE2 is development of the AAL Roadmap and Strategic Research Agenda. This paper shows the preliminary results of the AAL Roadmap developed up to now in the context of this project.

\section{AALIANCE2 and methods}

The aim of the AALIANCE2 Project is to study and investigate the technological innovations needed in the next years for the real deployment and exploitation of AAL services in the society.

The AAL Roadmap and the Strategic Research Agenda written during the Project will propose new potential and necessary AAL scenarios and the technologies that should be developed to provide these new services to elderly persons and to formal and informal caregivers. This work took as its starting point the AALIANCE AAL Roadmap, a document developed in 2010 during the AALIANCE Project [2].The workflow followed during the elaboration of the roadmap is shown in Figure 1.

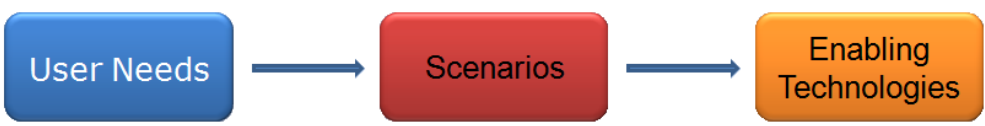

Figure 1. Stream used in the development of the AALIANCE2 AAL Roadmap and the SRA 
The first phase was devoted to the identification of actual necessities of senior persons and caregivers. Starting from this information new scenarios of AAL services, effective for satisfying real user needs, were conceived. Finally, the innovations and challenges for the five enabling technologies (acting, reasoning, sensing, communicating and interacting) necessary for developing the new AAL service scenarios were studied and presented. In the following paragraphs the main results of these three work stages reported in the first preliminary version of the AAL Roadmap are briefly presented.

\subsection{User needs}

The AAL Stakeholders were identified and divided into four groups, which are:

- Primary Stakeholders: old people and informal caregivers;

- Secondary Stakeholders: organisations offering services;

- Tertiary Stakeholders: organisations supplying goods and services;

- Quaternary Stakeholders: organisations related to the economic and legal context of AAL.

The identification of stakeholder necessities was the first step of the work and was necessary in order to conceive the key AAL service scenarios that were really implementable and effective in the society. These needs were identified starting from the experience of AALIANCE2 partners, representatives of the four kinds of stakeholders and an analysis of the state of the art. Moreover, some questionnaires were also developed and filled in by external experts in order to collect their opinions about the needs of AAL users and to find suitable solutions for all stakeholders. The choice of AAL solutions also took into consideration the AAL market, because the AALIANCE2 Project aims at suggesting services and systems that can be commercialized.

Analysing the elderly persons' point of view, it emerged that they want to maintain their independence, autonomy and dignity without, however, being excluded and uncared for by society. They want to remain in their own home, but at the same time they want to feel safe there; in other words, if something happens to them, they want to be able to easily get in contact with someone who can help them, or receive help directly from the "environment". Moreover, old persons are at high risk of being isolated, due to their weakening health and to territory. Indeed, Europe presents a variegated territory made of metropolitan district and wide rural areas. These geographical differences influence access to services and could then increase the isolation of older persons due to their increased difficulty in moving. 
Furthermore, the economic crisis strongly influences the lives of most of the elderly population increasing their risk of poverty and social exclusion - as shown in the Eurostat studies [3] - and consequently worsening their health and quality of life [4].

The AAL approach should help elderly people stay healthy as long as possible, allowing them to keep on living their own life and helping them to be involved in society and to continue working if they wish to. Senior subjects feel they are citizens who may still be useful in society, so they would like to be more included and involved in community life. This is important not only for them but also for society itself, which can still benefit from their experiences and capabilities.

Moreover, AAL should help informal caregivers in managing elderly people, reducing the stress and also the amount of work related to that. AAL devices would allow them to monitor older persons more easily, as well as to maintain contact with experts who could support them in making decisions and in evaluating the best behaviour to adopt. This kind of help could also be useful for formal caregivers. Often these professionals spend short periods of time with each of their senior users because they have to follow several people. If AAL devices could optimise their work, make it more efficient and also carry out part of it autonomously, they could spend more time with the elderly people and focus their efforts on more important aspects.

Furthermore, in analysing the needs of all stakeholders, the importance emerged of considering the economic and legal contexts, factors strongly influencing AAL exploitation and deployment in society. In particular, the economic crisis is having a negative influence on various countries, which are making cuts both to health care and to socio-medical services, while many older persons do not have the economic wherewithal to buy these servicesprivately. For these reasons it is important to identify both new AAL solutions that could reduce the costs of health and socio-medical carers, e.g. to reduce hospitalization, and also alternative forms of finance for these services.

\subsection{Service Scenario}

The scenarios described in the current version of the AALIANCE2 Roadmap are identified on the basis of three main service areas:

- Prevention, that is, action to reduce or eliminate the onset, causes, complications or recurrence of disease;

- Compensation and Support, which concerns elderly people with physical or cognitive impairments who need help in their daily activities; 
- Independent and Active Ageing, which aims to extend healthy life expectancy and Quality of Life for all people as they age, including those who are frail, disabled and in need of care.

These areas are at the same time complementary and overlapping, as shown in Figure 2.

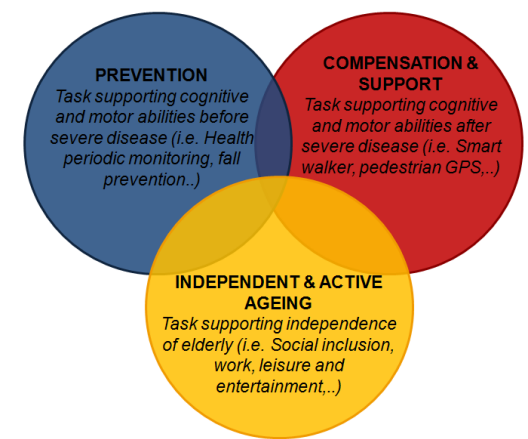

Figure 2. The AALIANCE2 macro-areas: Prevention, Compensation and Support, and Independent and Active Ageing

These aspects are strongly related to the Quality of Life (QoL) of senior citizens. Some research $[5,6]$ has asserted that the perception of QoL is conditioned mainly by five factors: (physical) health, (psychosocial) welfare, social contacts, activities and living environment. The way in which each of them influences QoL depends on personal experiences and life $[5,6]$. The three AALIANCE2 service areas are strongly correlated to these aspects; therefore, innovative AAL services related to these areas could positively influence the QoL of elderly people. Figure 3 shows how the QoL of elderly people could be influenced by Prevention, Compensation and Support and Independent and Active Ageing.

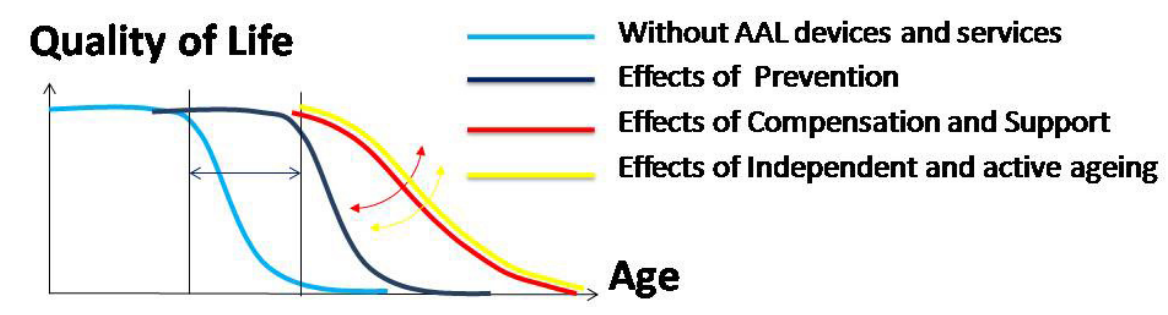


Figure3. Possible effects on Quality of Life

In particular, the light blue line of Figure 3 represents the QoL of a person who is not provided with any AAL technologies and services. It would decrease after a certain age due to disabilities and morbidity related to age. This line would decrease later (dark blue curve) if people perform a series of preventative actions that help delay or better managing diseases, in order to reduce or eliminate morbidity. When, coupled with prevention, compensation and support actions are carried out, the line could decrease even more slowly (red curve). As a matter of fact, ICT solutions could help patients do desired activities, making them perceive a higher QoL. Eventually, maintaining independence and remaining active, continuing to live their own life, increases the perceived Quality of Life (yellow curve).

Thanks to the workshops carried out during the AALIANCE2 Project, several AAL service scenarios were identified, describing some possible care services implementing ICT and robotic solutions to help elderly people in their daily life.

Some examples of scenarios are briefly listed, as follows:

- Healthy living: an intelligent kitchen suggesting recipes for healthy meals, also considering what is going to expire soon in the fridge;

- Falls: advanced environmental and wearable sensors linked to intelligent systems could prevent possible falls during daily activities;

- Prevention for health: motor and cognitive games carried out with smart tools to maintain elderly people's active and healthy lives, to support rehabilitation and prevent cognitive diseases;

- Senior citizens at work: considering the actual socioeconomic context, it is important to help people to keep working longer, and this can be done by supporting them in learning new skills and also in doing their daily work thanks to smart tools;

- Support in driving: smart cars proving more information about the traffic and street status and helping the elderly to drive safely;

- Sensing emotion: by understanding emotions, formal and informal caregivers could better understand how the elderly people are feeling and how best to help them;

- Assistance at home: a robotic assistant that helps older persons to carry out the most complex and dangerous activities of daily life in caring for him/herself and the house;

- Keeping social contacts: age-friendly environment that enables easier access to services, so to make people more involved in the society. Moreover radio and television can be used to help people interact with others and create topic discussion channels. 


\subsection{Enabling Technologies}

Starting from these scenarios, some technological components and innovations necessary for the development of these services were identified. AAL systems are typically composed of sensors, components that process the sensor data and derive conclusions, human-machine interface components and actors that execute all the actions. Finally all these components have to communicate with each other. These enabling technologies were then grouped into:

- $\quad$ Sensing

- Reasoning

- Acting

- Interacting

- Communicating.

Sensors are necessary in AAL systems in order to perceive what is happening. New sensors need to be developed in order to design new technologies that could improve elderly people and caregivers' life. New environmental sensors should be developed in order to reduce their impact in people's life. Besides vision sensors, a new generation of acoustic and smell sensors should be designed. These kind of sensors could in fact help informal and formal caregivers to take care of elderly people, by adding to the vision system other devices that could help them to understand what is going on. Moreover, it is important to improve the personal sensors, increasing acceptability and reducing invasiveness. That means that new wearable and implantable sensors should be developed and all these new sensors should be designed taking into account the challenge of power management and energy harvesting.

In order to deal with the great amount of data coming from different sensors, it is necessary to have good communication networks (both wired and wireless) and communication protocols. It is thus necessary to have communication standards and interoperability between systems and components. The main developments have to be made in the fields of body area or personal area network, local area or home network and wide area network, in order to allow communication between more systems and components. Furthermore, cloud computing is also considered a promising technology that could improve AAL services. In particular, it allows for managing data and supporting all the devices collaborating together. In this network of devices that can be useful for elderly people, robots must also be considered. Wearable robots and service robots could support elderly people in their lives, helping them feel more independent and safe.Moreover, cloud robotics is an enabling technology that integrates different agents together to improve collaboration and provide useful and high quality services to citizens. 
If all these AAL systems are to be part of everyday life, it is important that people can interact easily with them. Efforts have to be made to make this interaction "friendly" and usable to encourage users in using AAL systems. New interaction systems should be then be developed, based for example on spatial interfaces, such as 3D movement-tracker or gesture-based interaction, or on sensorial interfaces.

Furthermore people should feel safe with the new AAL systems, considering them as an advantage rather than an additional problem or cause of stress. For this reason systems should be dependable and should also be able to automatically manage any possible system failure, so to prevent damage or help the user to manage it. New challenges in process systems include the capability to recognize emergency situations as well as user habits, so as to identify uncommon situations. Moreover, the systems could be able to learn from the users' habits, so as to change with them and remain up to date with users' needs.

It is important to make people feel comfortable with the technologies. Acceptability is a fundamental aspect in AAL because it conditions the real use of these new devices by elderly persons and makes them feel the system is part of their home or themselves. Only by satisfying this precondition can AAL technologies can find a place in the real market and be commercialized.

In the end it is important to underline that technology should be an "enabler" something that helps elderly people feel better and helps formal and informal caregivers in their work; but technology per se is useless without the human components provided by the experience of the caregiver and from his/her relationship with older person. AAL systems should thus help to do time-consuming chores, allowing family and caregivers to spend more time with the elderly persons.

\section{Conclusion}

The AAL Roadmap developed in the first period of the AALIANCE2 Project aims at finding suitable new service and technological solutions in the AAL field, starting from user needs, that will allow old persons to remain healthy and active for as long as possible. In this way senior citizens can remain involved in society, continuing to be part of it and working for as long as they want. Moreover, one of the objectives of this project is the identification of challenges both for the AAL research and for the industry in the coming years.

This paper reports a brief summary of the first version of the AAL Roadmap. In the next months the AALIANCE2 AAL Roadmap and Strategic Research Agenda will be updated in order to refine the contents, considering new technologies and also feedback from the different experts and stakeholders involved during the project. 


\section{Acknowledge}

The research leading to these results has received funding from the European Community's Seventh Framework Programme (FP7/2007-2013) under grant agreement num. 288705 (AALIANCE2 Project).

\section{References}

1. Special Eurobarometer 378, (2012), "Active Ageing", http://ec.europa.eu/public opinion/archives/ebs/ebs 378 en.pdf

2. G. van der Broek, F. Cavallo, and C. Wehrmann, "AALIANCE Ambient Assisted Living Roadmap", Vol. 6, IOS Press, 2010.

3. Eurostat, "People at risk of poverty or social exclusion," 2013. [Online]. Available: http://epp.eurostat.ec.europa.eu/statistics_explained/index.php/People_at_risk_of_poverty_or_soc ial_exclusion.

4. AGE Platform Europe, "Older people also suffer because of the crisis", 2012.

5. Bowling A., Gabriel Z., Dykes J., DowdingLM., Evans O., Fleissig A., Sutton S. (2003). Let's ask them: A National Survey of Definitions of Quality of Life and its Enhancement Among People aged 65 and Over, Journal of Aging and Human Development, Vol. 56 (4), 269-306.

6. Puts M.T.E., Shekary N., Widdershoven G., Heldens J., Lips P., Deeg D.J.H. (2007). What does Quality of Life Mean to Older Frail and Non Frail Community-dwelling Adults in the Netherlands?, Journal of Quality of Life Research, Vol. 16, 263-277, doi: 10/1007/s11136-0069121-0 